\title{
Der Umbau ist möglich
}

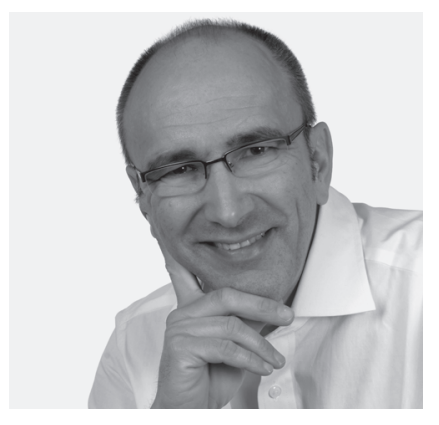

VON STEFAN BLANK

Stefan Blank ist Geschäftsführer des "PsychoSozialen Netzwerks" in Ludwigsburg. Als erster Mitarbeiter des im Jahre 1989 gegründeten Trägervereins ist er mit Entwicklungschancen und der Organisationsentwicklung gemeinnütziger Einrichtungen langjährig vertraut. Seine Arbeitsschwerpunkte liegen im strategischen Management und in der Umsetzung personenzentriert und sozialräumlich wirksamer Versorgungskulturen in der Sozialpsychiatrie.

E-Mailinfo@reifezeit.eu

\author{
In einigen Geschäftsfeldern der Sozialwirtschaft ist der \\ Wechsel von einer einrichtungszentrierten Versorgung \\ (»Wo gibt es freie Plätze?») zu einer personenorientierten \\ Betrachtung ("Was braucht dieser Mensch mit seinen \\ spezifischen Ressourcen an zusätzlichen Hilfen?») in \\ vollem Gange. Das "Ludwigburger Modells" zeigt \\ am Beispiel der Gemeindepsychiatrie, wie dieser \\ grundlegende Wandel politisch, konzeptionell und \\ betriebswirtschaftlich organisiert werden kann.
}

Die inhaltliche Reform der Eingliederungshilfe ist ins Stocken geraten. Bund und Länder schweigen, wo es doch um alle Menschen mit Behinderungen und eine zukünftig inklusive Gesellschaft geht. Die Hoffnung der Freien Wohlfahrtspflege, dass die entsprechenden sozialrechtlichen Rahmenbedingungen noch in dieser Legislaturperiode geschaffen werden könnten, um Menschen mit Behinderung das Leben und Arbeiten - dort, wo Sie es wollen - künftig zu erleichtern, dürfte sich nicht erfüllen.

Möglicherweise sind wir jetzt in der Sozialwirtschaft an einem Wendepunkt angekommen, an dem wir zunächst eigene Barrieren überwinden müssen, ehe wir den richtigen Weg wählen können. Ob wir die Zeit noch haben, die es braucht, lässt sich im Hinblick auf globale, kaum beherrschbare finanzwirtschaftliche Kräfte nicht unbedingt mit Ja beantworten.

\section{Der Entwicklungsweg einer institutionszentrierten Versorgungslogik}

Alles deutet darauf hin, dass die einrichtungszentrierten Entwicklungswege, dem Pendel einer Standuhr gleich, an einem Wendepunkt angekommen sind, kurz zum Stillstand kommen, ehe der Pendel in die andere Richtung zu- rückschlägt. Schon Konfuzius suchte Erklärungen für solche Umwälzungen. Wir folgen seinem Rat und führen uns am Beispiel der Stadt Ludwigsburg im heutigen Baden-Württemberg »das Alte noch einmal vor Augen, um das Neue zu erkennen $«$.

- Das Tollhaus 1749: In Ludwigsburg entstand das erste »Tollhaus « in Württemberg. Mitten in der Stadt diente es der Verwahrung »der Tollen ", später der Behandlung der »Irren«. Anfang des 18. Jahrhunderts entsprach das Raumprogramm nicht mehr den Anforderungen einer zeitgemäßen ärztlichen Behandlung. Das Stuttgarter »Medicinalcollegium» empfahl dem König von Württemberg, das Ludwigsburger Tollhaus aufzulösen und dafür das leerstehende Kloster in Zwiefalten so umzubauen, dass 93 Einzelzellen, Konversationsräume und Bäder eine zeitgemäße Behandlung ermöglichen. Der König war zwar einverstanden, aus finanziellen Gründen mussten die Umbaupläne aber verworfen werden.

- Ausgangspunkt der Institutionszentrierung 1812: Trotz der finanziellen Restriktionen wurde am 21. August 1812 »das hiesige Irreninstitut mit dem letzten Irrentransport in das neue Irrenhaus nach Zwiefalten versetzt $«$. 


\begin{tabular}{|c|c|c|c|}
\hline & Privatsektor & Dritter Sektor & Öffentlicher Sektor \\
\hline Rechtsform & $\begin{array}{l}\mathrm{GmbH}, \mathrm{AG}, \mathrm{KG}, \mathrm{OHG} \text {, } \\
\text { etc. }\end{array}$ & $\begin{array}{l}\text { Stiftung, Verein, Genos- } \\
\text { senschaft }\end{array}$ & $\begin{array}{l}\text { Öffentliches Unterneh- } \\
\text { men }\end{array}$ \\
\hline Charakteristikum & Profitgebot & Ausschüttungsverbot & $\begin{array}{l}\text { Gleichberechtigungs- } \\
\text { gebot }\end{array}$ \\
\hline $\begin{array}{l}\text { Finanzierungs- } \\
\text { quellen }\end{array}$ & $\begin{array}{l}\text { Verkaufseinnahmen, } \\
\text { Kredit- und Kapitalmarkt }\end{array}$ & $\begin{array}{l}\text { Öffentliche Zuschüsse, } \\
\text { Gebühren, Spenden }\end{array}$ & $\begin{array}{l}\text { Gebühren, Öffentliche } \\
\text { Gelder }\end{array}$ \\
\hline $\begin{array}{l}\text { Koordinations- } \\
\text { mechanismus }\end{array}$ & Wettbewerb & Kooperation & Hierarchie \\
\hline
\end{tabular}

Abb. 1: Jeder Zone wirtschaftlicher Betätigung hat ihre eigenen charakteristischen Merkmale.

Quelle: Charles B. Blankart und Björn Gehrmann, Der Dritte Sektor in der Europäischen Union: Die Daseinsvorsorge aus ökonomischer Sicht.

Das Ende der örtlichen Irrenfürsorge ist gleichzeitig der Ausgangspunkt einer institutionszentrierten Entwicklung. Während die Ärzteschaft an besseren Behandlungsmöglichkeiten interessiert war, sah die königliche Verwaltung in der Zentralisierung die Möglichkeit, funktionale Prozesse direktiv und ökonomisch zentral zu steuern. Diesem unteilbaren, ökonomischen und organisatorischen Prinzip zentralisierter Prozesse sind Verwaltungen im öffentlichen Sektor der Daseinsfürsorge (Abb. 1) bis heute unterworfen. Ihr Ziel ist, mit möglichst wenig Aufwand, einen maximalen Nutzen zu erreichen. Auf der
Grundlage der überregionalen institutionszentrierten Versorgungslogik war der Bau weiterer Heilanstalten aufgrund steigender Fallzahlen notwendig.

- Die erste Chance für den Wandel 1895: Dreißig Bürger, darunter Anstaltspsychiater, einige höhere $\mathrm{Be}$ amte und Geistliche, wurden auf die drohende Fehlentwicklung aufmerksam und gründeten im Jahre 1895 den »Hilfsverein für reconvalescente Geisteskranke in Württemberg «. Erstaunlich war, dass sie bereits vor über hundert Jahren das Ziel formulierten, die »Irrenfürsorge außerhalb der Anstalten" aufzubauen. Sie hiel- ten es für besser, die Rekonvaleszenten in der Mitte der Gesellschaft im Auge zu behalten, anstatt ihnen in den großen Anstalten jede Perspektive auf Normalität zu nehmen. Denkbar war für sie, auf ein förderliches Verhalten der Umwelt hinwirken zu können. Mit heutigen Worten würden wir sagen, ihr Ziel sei die Integration, die sozialräumliche Vernetzung und Inklusion gewesen. Bis heute nicht gelöst ein weiteres Ziel: die Vermittlung der Rekonvaleszenten in geeignete Arbeitsstellen. Die Feldstrukturen der Aufmerksamkeit (Abb. 2) von ClausOtto Scharmer tragen dazu bei, die wirksamen Kräfte der Koordination und der Macht in vier Entwicklungsphasen identifizieren zu können. Im Falle des Hilfsvereins handelt es sich um eine frühe Netzwerkstruktur. Scharmers Phasenmodell über $»$ die Evolution institutioneller Feldstrukturen « (Abb. 3) beschreibt die phasentypischen Aktivitäten. So handelt es sich beim Austausch der dreißig Vereinsmitglieder um »StakeholderProzesse « und in der Aufmerksamkeit einander zuzuhören oder sich mitzuteilen um »Stakeholder-Dialoge«. Diese wechselseitigen Entwicklungen auf der Beziehungsebene bilden die Grundlage für Netzwerke mit innovativen Potenzialen für neue Organisationsformen (»Eco-System«). In
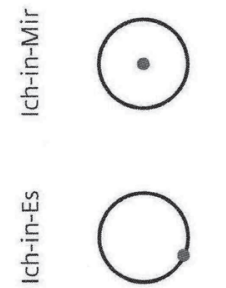

\section{Zentralisierung:}

Maschinenbürokratie

2

Dezentralisierung:

Divisionen

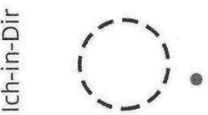

3

Netzwerk:

Matrix

4

Eco-Systems der Innovation
Machtquelle: Hierarchie

zentralisierten Regeln folgen zentrumgetrieben

Logik: Ökonomie der Skalenerträge (Produktion)

Machtquelle: Markterfolg

Marktnachfrage befriedigen peripheriegetrieben

Logik: Ökonomie der Kundenbeziehungen

Machtquelle: vernetzte Beziehungen

Netzwerke mobilisieren beziehungsgetrieben

Logik: Ökonomie der Innovation (Produktinnovation)

Machtquelle: Feld der enstehenden Möglichkeiten Eco-Systems der Innovation umfeldgetrieben Logik: Ökonomie des Presencings (Feldinnovation)

Vier Typen der Koordination - vier Geometrien der Macht

Abb. 2.: Vier Entwicklungsphasen können als wirksame Kräfte der Koordination und der Macht identifiziert werden.

Quelle: Claus Otto Scharmer, Theorie U. Von der Zukunft her Führen. Presencing als soziale Technik. 


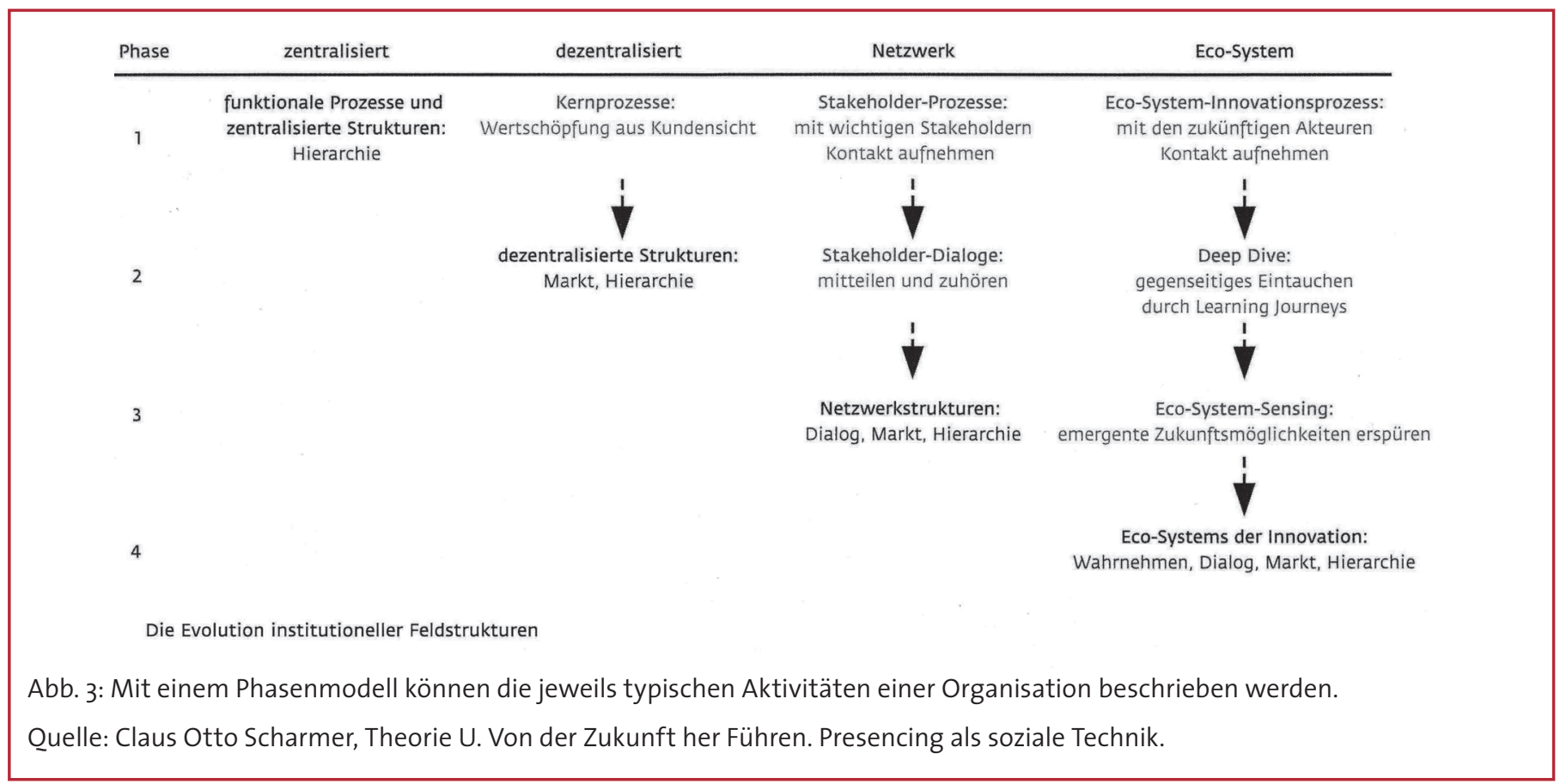

zu sorgen und die notwendige Koordination aller Versorgungsdienste zu gewährleisten. Darüber hinaus hielten sie den Auf- und Ausbau eines bedarfsgerechten, gemeindenahen Versorgungssystems mit ambulanten und komplementären Diensten und koordinierten Versorgungssystemen innerhalb der Standardversorgungsgebiete für notwendig. Die Psychiatrie-Enquete leitet den Übergang ein in die Entwicklungsphase der Dezentralisierung.

- Die Dezentralisierung 1979: Auch die Bürger aus dem Landkreis Ludwigsburg profitierten von der Psychiatrie-Enquete. Der Aufbau einer psychiatrischen Abteilung am örtlichen Kreiskrankenhaus wurde möglich. Erstmals, nach 167 Jahren, konnten wieder psychisch kranke Bürger innerhalb des Landkreises akut behandelt werden. Gleichzeitig musste die Expertenkommission weitere sechs Jahren mit den Kostenträgern über die Finanzierung notwendiger ambulanter Dienste verhandeln. Die Phasenentwicklung der Dezentralisierung lässt sich mit der Entwicklung von Einrichtungen der Freien Wohlfahrtspflege von $1.500 \mathrm{im}$ Jahr 1970 auf $15.000 \mathrm{im}$ Jahr 2008 gut belegen.

- Gründung eines Fördervereins 1989: Der noch jungen psychiatrischen Klinik in Ludwigsburg fehlten die stabilisierenden Nachsorgeeinrichtungen in den Gemeinden völlig, um der Übersicht der drei Sektoren der Daseinsfürsorge werden die Ausgangslage, die trennenden Barrieren und Interessen zwischen profitorientierten Pflegefabriken im Privatsektor, gemeinnützige Einrichtungen im dritten Sektor und dem öffentlichen Sektor sichtbar. In welchem der drei Sektoren wird Teilhabe für Menschen mit Behinderung in der Mitte der Gesellschaft zukünftig am nachhaltigsten und wo am wirtschaftlichsten $\mathrm{zu}$ entwickeln sein?

- Die Risiken und Folgen verpasster Chancen 1895: Während dreißig Gründungsmitglieder ein radikal neues und innovatives Fürsorgeverständnis entwickeln, reift auch eine andere Idee heran, die mit der Schrift über die »Grundlinien einer Rassenhygiene « in der Öffentlichkeit auf sich aufmerksam macht. Nachdem unter schwierigsten wirtschaftlichen, humanitären und ökonomischen Rahmenbedingungen im Umfeld zweier Weltkriege, zunächst Zehntausende behinderte Menschen in Anstalten verhungert sind, beschäftigt sich eine weitere Schrift im Jahr 1920 mit einer Rechtskonstruktion der »Freigabe der Vernichtung lebensunwerten Lebens", was mit der Machtergreifung Hitlers im Jahre 1933 wesentlich zu einem Gesetz beiträgt, das die Zwangssterilisation von insgesamt 400.000 Menschen zur Folge hat. Zuletzt, in der Zeit zwischen 1939 und 1944, mündet diese Entwicklung in der Ermordung von 70.000 Psychiatrie-Patienten.

- Die Psychiatrie-Enquete 1975: Der nationale Irrweg hat sich tief ins kollektive Unterbewusstsein unserer Gesellschaft eingegraben, anders ist nicht zu erklären, dass Menschen mit Behinderung zwischen 1945 und 1970 weiterhin in großen Anstalten und in Schlafsälen mit zehn, zwanzig oder dreißig Betten leben mussten. Der Berufsgruppe psychiatrisch Tätiger, dem »Mannheimer Kreis «, ist es zu verdanken, dass die prekären Versorgungszustände in psychiatrischen Groß- und Komplexeinrichtungen am Anfang der 1970er Jahre öffentlich zur Sprache gebracht wurden. Die Netzwerkaktivitäten führten zur Einsetzung einer Expertenkommission durch den Deutschen Bundestag. Die Öffentlichkeit war schockiert, was die Umkehr sozialpolitischen Denkens und Handelns beschleunigte und zeitnah zum Aufbau dezentraler psychiatrischen Klinken und Abteilungen führte. Das wichtigste Ziel der Psychiatriereform, die Auflösung der psychiatrischen Groß-und Komplexleistungsträger, scheiterte zwar am Widerstand dieser Institutionen. Dennoch ist die Psychiatrie-Enquete eine bestimmende Kraft geworden. Ihre wichtigsten Empfehlungen waren, für eine bedarfsgerechte Versorgung aller psychisch Kranken und Behinderten 


\section{Inklusion - ein anderer Blick auf die Welt}

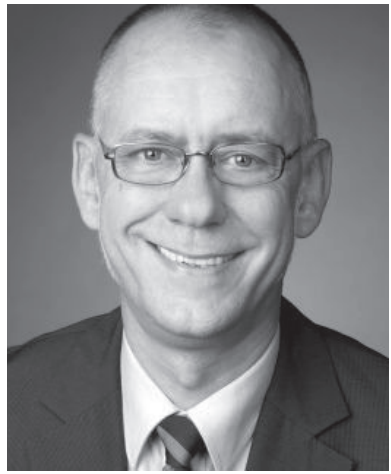

Mit dem Sozialgesetzbuch IX, dem SGB $X I I$, dem Bundesgleichstellungsgesetz und der Ratifizierung der Behindertenrechtskonvention setzt sich in Deutschland seit nunmehr über zehn Jahren ein Wandel im gesellschaftlichen Miteinander behinderter und nicht behinderter Menschen in Bewegung, der vielleicht mit der Entwicklung der Emanzipationsbewegung in den 7oer Jahren des vorigen Jahrhunderts vergleichbar ist.

Ein solcher Wandel braucht seine Zeit, aber er ist vergleichbar mit einem Zug der inzwischen Fahrt aufgenommen hat und nicht mehr zu stoppen ist. Da hilft es wenig, die Errungenschaften und die Bedeutung bisherigen Handelns zu begründen. Vielmehr werden es künftig die bisherigen Objekte sozialpädagogischen Handelns sein, die als Subjekte selbstbestimmt(er) ihr Leben in die Hand nehmen. Dies mag heute noch unvorstellbar anmuten, wie es früher vielen Männern noch undenkbar erschien, dass ihre Ehefrauen auf einmal ihren Arbeitsvertrag selbst unterschreiben können.

Der mit der UN-Behindertenrechtskonvention aufgezeigte Weg zeichnet neue Konturen in der deutschen Landschaft der Behindertenhilfe. So haben die von den Wohlfahrtsverbänden mit getragenen Soziallotterien ihre Förderung in den letzten zwei Jahren neu ausgerichtet:

- Die Aktion Mensch fördert besonders regionale Projekte gemeinnütziger Organisationen, die mit nicht gemeinnützigen Partnern kooperieren, um das Wohnen, das Arbeiten, die Freizeit oder die Barrierefreiheit behinderter Menschen mit den vorhandenen Angeboten in der Gesellschaft zu vernetzen.

- Die Stiftung deutsches Hilfswerk unterstützt gemeinnützige Träger bei der Schaffung sozialraumorientierter Quartierskonzepte für ältere, pflegebedürftige Menschen.

Zugleich ist der Ausstieg aus der Förderung von Sondereinrichtungen wie Werkstätten, Sonderkindergärten und Sonderschulen bei der Aktion Mensch Realität und das Deutsche Hilfswerk unterstützt den Bau klassischer ambulant betreuter Wohnangebote für ältere Menschen nur noch unter sehr engen Grenzen. Ziel ist, das Lebensumfeld von Menschen so zu gestalten, dass sie mehr Orientierung bieten und Menschen mit Hilfe- und Pflegebedarf länger zu Hause wohnen und leben können.

Der Fokus liegt auf gesellschaftlicher Teilhabe, Selbstbestimmung und barrierefreier Gestaltung aller Lebensbereiche. Menschen mit Behinderung sollen stärker als bisher in der Gemeinde und im Stadtteil als Akteure und Teilhabende sichtbar werden. Hierzu sollen gemeindeintegrierte Wohn-, Betreuungs- und Freizeitangebote geschaffen und gestärkt werden.

Die Schneise, für die auch Fördermittel für diese noch nicht refinanzierbaren Aufgaben zur Verfügung stehen, ist bei den Soziallotterien geschlagen, es fehlt manchmal noch an der zündenden Idee und am Mut, mit neuen, kleinräumigen Projekten die Schneise als Weg auszugestalten.

\section{Joachim Hagelskamp}

Joachim Hagelskamp ist beim Gesamtverband des Paritätischen Wohlfahrtsverbandes in Berlin zuständig für die wirtschaftliche Förderung der bundesweit rund 10.000 Mitgliedsorganisationen des Verbandes.

E-Mail blf@paritaet.org den Behandlungserfolg sicherstellen und den »Drehtüreffekt« verhindern zu können. Allein mit der Möglichkeit einer wohnortnahen psychiatrischen Behandlung lässt sich ein gesellschaftliches Bewusstsein für die Notwendigkeit der Integration eines eineinhalb Jahrhunderte lang abwesenden Personenkreises nicht entwickeln. Der »blinde Fleck« machte es möglich, sich im Verlauf von 194 Jahren daran zu gewöhnen, dass Bürger des Landkreises bei Bedarf in Pflege-und Eingliederungsheime anderer Landkreise verlegt werden. Bei einer überregionalen Versorgungslogik gibt es keinen Grund, dezentrale stationäre Wohn-und Betreuungseinrichtungen in der Eingliederungshilfe in allen Landkreisen aufzubauen. Der Initiative des damaligen Chefarztes ist es zu verdanken, dass die Gründung eines Fördervereins möglich wurde, in dem alle wichtigen Stakeholder der Region sowie Privatpersonen, Betroffene und Angehörige als Gründungsmitglied vertreten waren. Erneut waren es Stakeholder-Dialoge in Netzwerkstrukturen, die Entwicklungen ermöglichten.

- Die Chance örtlicher Zuständigkeit 2005: Der Entwicklung des Ludwigsburger Modells wäre ohne die Auflösung der überörtliche Sozialhilfeträger in Baden-Württemberg nicht zustande gekommen. Mit der $\mathrm{Zu}$ ständigkeit der 44 Stadt- und Landkreise schien es erstmals möglich, in regional neu zu schaffenden Versorgungsstrukturen und in örtlicher Selbstbestimmung, eine zeitgemäße sozialraum-und personenzentrierte Versorgungslogik entwickeln zu können. Wir selbst gingen davon aus, im dritten Sektor der öffentlichen Daseinsfürsorge unterwegs zu sein und erwarteten, dass sich Chancen, durch die Initiative des Daseinsverantwortlichen, dem Landkreis, ergeben würden. Wir mussten recht früh feststellen, dass es der neue örtliche Kostenträger anders sieht. Wir waren mitten im Privatsektor angekommen, in dem der örtliche Kostenträger den Wettbewerb nach seinen Regeln bestimmt. Unsere Entwicklungsperspektive führte über die Konkurrenz unter Trägern. Gegenseitige Verletzungen und der Verlust unserer sozialräumlichen Kooperationsfähigkeit, aber 
auch eine abnehmende öffentliche Akzeptanz sind die Folgen. Möglicherweise würden wir Menschen mit Behinderung nur noch als Markt begreifen, den es zu verteidigen oder zu erobern gilt. Hinter uns lagen bereits Jahre des Entwicklungsstillstands in überörtlicher Sozialhilfeträgerschaft, doch mit der örtlichen Sozialhilfeträgerschaft wurden plötzlich unerwartet starke, zentrumsgetriebene Kräfte frei, die uns aus dem Gleichgewicht brachten. Unsere Ziele, unser Selbstverständnis und unsere Unternehmenskultur sind in den Sog dieser zentrumsgetriebenen Kräfte geraten, einer Entwicklungsrichtung, der wir nichts entgegenzusetzen hatten. Erst das Begreifen, dass es hier nichts mehr zu verlieren gibt, setzte neue Kräfte frei. Wir führten Bedarfserhebungen für stationäre Platzzahlen durch, suchten immer wieder das Gespräch und stellten erfolglos Anträge für stationäre Plätze. Eine Entscheidung fiel schließlich in einem Spitzengespräch, was zur Bedarfsfeststellung von 24 Wohnheimplätzen führte. Gleichzeitig suchten wir den Kontakt zu den Konkurrenten, eröffneten den konstruktiven Dialog, schalteten Moderatoren ein, um uns gegenseitige die Angst voreinander zu nehmen. Mit diesem Schritt haben

\section{Das Ludwigsburger Modell}

will jedem Menschen genau die Hilfe bekommen zukommen lassen, die er benötigt - das ist das Ziel des Projekts »Flexible Hilfen«. Der Landkreis Ludwigsburg und die Träger der Freien Wohlfahrtspflege im Landkreis haben das Projekt ins Leben gerufen, um verschiedene neue Betreuungsmodelle auszuprobieren. Wo es bisher nur die Wahl gab, zwischen ambulant (= eher wenig Hilfe) und stationär (= Wohnheim), soll es jetzt Zwischenstufen geben für alle die, die zwar ein gewisses Maß an Hilfe benötigen, aber dennoch nicht in einem Wohnheim leben möchten. Auch die PsychoSoziales Netzwerk gGmbH erprobt entsprechende flexible Modelle für Menschen mit psychischen Erkrankungen im Rahmen dieses Projekts. Möglich sind beispielsweise die Varianten: Betreutes Wohnen in der eigenen Wohnung, Betreuung in einer Wohngemeinschaft, Betreuung im Apartment. Das Projekt läuft noch bis Ende 2012; es wird wissenschaftlich begleitet und ausgewertet.

www.psn-lb.de

www.landkreis-ludwigsburg.de

mulierten wir ein gleichlautendes Schreiben, das jeder Träger an den Landrat richtete. Es war die Einladung, sich im dritten Sektor zu begegnen, verbunden mit dem Wunsch zur Kooperation, um gemeinsam an der Entwicklung personenzentrierter, durchlässiger und flexibler Hilfen arbeiten zu können. Erst jetzt öffnete sich die Tür - und sie blieb offen. Die wichtigsten Stakeholder mit unterschiedlichen Aufgaben und Rollen arbeiten bis heute verbindlich zusammen. Erneut finden sich Merkmale

\section{"Aus 24 stationären Heimplätzen wurden 21.000 Stunden ambulanter Leistungszeitmenge"}

wir den Privatsektor wieder verlassen können. Was wir aus der Not heraus in Gang setzten, nahm bald viel Zeit in Anspruch, um verlässliche Kooperationen zu allen Trägern aufzubauen und zu erhalten. Die Gründung eines Fachausschusses für Eingliederungshilfe, dem alle zwölf örtlichen Eingliederungshilfeträger angehörten, war ein wichtiger Entwicklungsschritt. In Arbeitsgruppen bildeten wir Schnittmengen aus individuellen Problembeschreibungen, die wir in einem Eckpunktepapier bündelten und gemeinsam dem Landkreis vorstellten. Doch nichts veränderte sich. Ende 2008 fassten wir den Mut zu einer neuen Initiative. Diesmal for- einer Netzwerkstruktur und die Entwicklung führte jetzt auch zu einem trägerübergreifenden Modellprojekt »Flexible Hilfen « parallel zu unserem Ludwigsburger Modell.

- Die nationale Chance der UN-Konvention über die Rechte von Menschen mit Behinderungen (BRK) 2009: Wir blicken in Ludwigsburg auf zweihundert Jahre eines institutionszentrierten Entwicklungswegs zurück. Ein langer Zeitraum, der vor allem von Vorstellungen der Kostenträger und großer Komplexleistungsträger geprägt war. Zwei, die sich immer darüber verständigt haben, was ein Dritter braucht, um behinderungsbedingte Nachteile gut ausgleichen zu können. Legitimiert durch die UN-Behindertenrechtskonvention erschient vor zwei Jahren erstmals der benachteiligte Personenkreis, in eigener Sache. Ihm war es in der zurückliegenden Zeit nicht möglich, Wünsche oder Wahlmöglichkeiten entwickeln zu können. Menschen mit Behinderung brauchen dabei jetzt unsere Unterstützung, um wünschen zu lernen, damit Wahlmöglichkeiten entstehen. In transparenten Hilfeplanverfahren werden individuelle Wünsche zur Planungsgrundlage. Sie sind als Teilhabeziele zu berücksichtigen. Gemeinsam sind wir für die Unterstützung verantwortlich, dass diese persönlichen Lebensziele entweder in stationären Einrichtungen oder inmitten der Gesellschaft verwirklicht werden können. Die betroffenen Menschen sind schon jetzt Herr des Verfahrens, wenn es um die Bestimmung des eigenen Wohnorts oder um den subjektiven Wunsch nach einer sinnvollen Beschäftigung geht. Doch noch sind die Kräfte der UN-Behindertenrechtskonvention nicht völlig freigesetzt.

- Regierungswechsel und neues Denken in der Landespolitik 2011: Wesentliche Entwicklungsimpulse sind in Baden-Württemberg von der neuen Landesregierung zu erwarten. Auch die Zielsetzungen des Koalititonsvertrages von Grünen und SPD deuten eine neue Entwicklungsrichtung an. Manche Ziele - wie die Schaffung eines Psychiatriegesetzes und die Herstellung eines verlässlichen, auf- 
einander abgestimmten Systems der komplementären Eingliederungshilfeleistungen - stimmen positiv. Die Grundlage eines transparenten und zeitgemäßen Bedarfsbemessungsinstruments zur Ermittlung individueller Unterstützungsbedarfe von Menschen mit Behinderung sollen so hergestellt werden, dass der individuelle Bedarf unabhängig von der institutionellen Umsetzung ermittelt und erbracht werden kann. Darüber hinaus soll ein Landesbehindertengleichstellungsgesetz grundlegend überarbeitet und mit einklagbaren Rechten ausgestattet werden.

\section{Das Ludwigsburger Modell: Zeit statt Plätze}

Auf der Grundlage einer neuen Verständigungskultur und der UN-Behindertenrechtskonvention haben wir uns gemeinsam mit dem Landkreis für einen Transformationsprozess entschieden, der von " 24 stationären Wohnheimplätzen« zu einer »sozialraumfähigen zeitbasierten Versorgungslogik « führen muss.

Unser Anspruch war, dabei alle Qualitäten stationärer Einrichtungen nachteilsfrei zu reproduzieren. Die Unteilbarkeit stationärer Plätze und die ausgrenzende Wirkung investiver Ausgleichsabgabefördermittel mussten überwunden werden, um an jedem Ort und im jeweils erforderlichen Umfang die Assistenz zur Rückkehr von Menschen mit Behinderung in die Mitte der Gesellschaft jederzeit gewährleisten zu können.

Folglich war die Teilbarkeit aller Vergütungen aus individuellen personenzentrierten Leistungen - aber auch alle Kosten einschließlich der Gebäuderefinanzierungen - zu bewerkstelligen. Die Leistungen, die in einer stationären Einrichtung mit 24 Plätzen erbracht werden konnten, mussten sich in einer teilbaren "Leistungszeitmenge « wiederfinden.

Wir legten die gewohnten Personalschlüssel zugrunde. Die ermittelten Jahresarbeitszeiten der neuen Organisation bereinigten wir um alle indirekten $\mathrm{Ab}$ wesenheits- oder Organisationszeiten, die dem Leistungsberechtigten nicht direkt zur Verfügung stehen. Auf diese Weise konnte ein neuer Leistungsfaktor von 21.000 Stunden allen Planungen zugrunde gelegt und der stationäre Platzfaktor ersetzt werden.
Die Jahreserträge der neuen Organisationsform sind nicht geringer als die Erträge einer Wohnstätte mit 24 Plätzen auf der Grundlage der vertrauten Vergütungssätze. Dieses Teilungsprinzip legten wir bei der Raumplanung zugrunde. Eine Apartmentanlage für 24 Mieter ermöglicht jetzt Wohnen für jeden. Eine auskömmliche Miete ist vereinbart worden und deckt den Schuldendienst.

Gleichzeitig sind aus 24 Heimbewohnern jetzt 24 Mieter mit allen sozialrechtlichen Leistungsansprüchen geworden. Im »Komplexleistungszentrum « entstanden abgeschlossene Raumeinheiten für einen selbstständigen psychiatrischen Pflegedienst und eine Ergotherapiepraxis; daneben Büroräume, Freizeit und Aufenthaltsmöglichkeiten. Auch die Gastronomie und der hauswirtschaftliche Trainingsbereich können im Eigenbetrieb entwickelt oder verpachtet werden.

Die Refinanzierung des Komplexleistungszentrums gelingt durch einen Investitionskostenzuschlag auf alle 21.000 Stunden. Führen individuelle Betreuungserfolge zur Absenkung der Leistungen, so erfolgt die Auffüllung der Leistungsmenge durch die Zuweisung weiter Leistungsberechtigter. Sieben Leistungsgruppen entsprechen vergleichbare Personalschlüssel von 1:1,5 bis 1:12. Die Teilbarkeit der Eingliederungshilfeleistung und ein in Euro pro Stunde hinterlegter Gegenwert ermöglicht Assistenzleistungen alleine oder koordiniert mit Dritten an jedem Ort im Landkreis. Braucht es weitere Partner im Nahbereich des Leistungsberechtigten, dann lassen sich Leistungszeiten teilen und im Interesse einer gemeinsame Versorgung koordinieren. Versorgungssicherheit trotz hoher Komplexität in den Sozialräumen ist möglich, weil nicht nur die Leistungen, sondern auch die Verantwortung für die Eingliederung auf mehr als nur einer Schultern ruht.

Wir stehen erst am Anfang einer Entwicklung; doch die Voraussetzungen sind hoffnungsvoll. Eine erfolgreiche Entwicklung, so hoffen wir, sollte sich in einigen Jahren auch daran ablesen lassen, dass deutschlandweit mehr als nur 360 Trägerübergreifende Persönliche Budgets im Verhältnis zu 730.000 Hilfeempfängern der Eingliederungshilfe zustande gekommen sind.

\section{"Standardwerk"}

Roland Wolf, SAE 1/05, zur Vorauflage

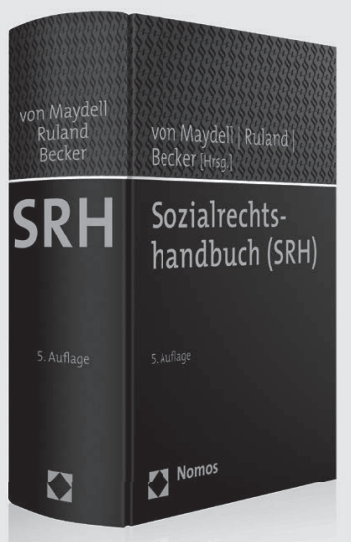

Sozialrechtshandbuch (SRH)

Herausgegeben von

Bernd Baron von Maydell,

Franz Ruland und Ulrich Becker

5. Auflage 2012, 1.625 S., geb., 158,-€ ISBN 978-3-8329-6462-7

www.nomos-shop.de/13448

Die Neuauflage des zum Markenzeichen gewordenen SRH bringt Sie auf den aktuellen Stand der Gesetzgebung und Rechtsprechung in allen wichtigen Sozialrechtsgebieten.

\section{Berücksichtigt werden u.a.:}

- die Gesetzesänderungen durch die jüngsten Gesundheitsreformen,

- die neuesten gesetzlichen Entwicklungen in der Rentenversicherung,

- die aktuellen Entscheidungen des Bundessozialgerichts, der Instanzgerichte und des Europäischen Gerichtshofs.

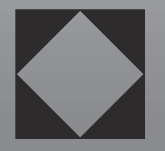

